\title{
Reduced levels of interleukin-1 receptor antagonist act as a marker for pneumonia in the elderly
}

\author{
LIJIE ZHAO ${ }^{1}$, LINA WANG ${ }^{1}$, XIAOYAN ZHANG ${ }^{1}$, LILI SUN $^{1}$, DAN SUN $^{1}$ and TINGLI SUN ${ }^{2}$ \\ Departments of ${ }^{1}$ Geriatrics and ${ }^{2}$ Nephrology, General Hospital of Daqing Oil Field, Daqing, Heilongjiang 163311, P.R. China
}

Received September 21, 2013; Accepted April 14, 2014

DOI: $10.3892 / \mathrm{mmr} .2014 .2284$

\begin{abstract}
Pneumonia is a disease causing serious inflammation and infection of the lungs and accounts for $>50,000$ mortalities annually. The elderly are at an increased risk of developing pneumonia. Pneumonia is more serious in the elderly than in any other age group due to the increased inflammation and risk of community-acquired pneumonia associated with aging. Interleukin-1 receptor antagonist (IL-1RA) is an anti-inflammatory protein that counteracts the destructive effects of inflammatory proteins. Therefore, the possible association between pneumonia in elderly individuals and reduced levels of IL-1RA was investigated in the present study. The number of lymphocytes was counted in all subjects and the relative protein expression levels of IL-1RA were determined using western blot analysis. In addition, the immunological activities of IL-1RA were measured using ELISA. The results demonstrated that the numbers of lymphocytes in the serum and bronchoalveolar lavage fluid (BALF) were significantly higher in elderly patients than those in young patients. Furthermore, the serum and BALF levels of IL-1RA in elderly patients were significantly lower than those in young patients $(\mathrm{P}<0.05$ and $\mathrm{P}<0.01$, respectively). Therefore, reduced levels of IL-1RA in BALF may act as a marker for pneumonia in the elderly and may be a potential adjuvant for the diagnosis of pneumonia in elderly individuals. The results also showed that smoking was associated with significant reductions in the levels of IL-1RA in the BALF of elderly patients. The association between smokers and non-smokers found in this study provides support for the hypothesis that smoking may contribute to the pathogenesis of pneumonia by further reducing IL-IRA levels in certain elderly patients.
\end{abstract}

Correspondence to: Professor Tingli Sun, Department of Nephrology, General Hospital of Daqing Oil Field, 9 Zhongkang Street, Daqing, Heilongjiang 163311, P.R. China

E-mail: tinglidq@163.com

Key words: bronchoalveolar lavage fluid, interleukin-1 receptor antagonist, pneumonia in the elderly, pneumonia in the young, lymphocytosis

\section{Introduction}

Pneumonia is a disease causing serious inflammation and infection of the lungs and represents one of the leading causes of mortality, accounting for $>50,000$ deaths annually (1). Individuals aged $\geq 65$ years are particularly vulnerable to developing the disease. Furthermore, pneumonia is more serious in the elderly than in any other age group (2). However, the diagnosis of pneumonia in older populations can be difficult since the elderly are less likely to report the symptoms associated with the disease, such as fevers, chills and chest pains. Chest radiography and physical examinations are necessary for a diagnosis to be obtained, but the diagnosis often remains difficult due to the fact that the accuracy of chest radiographs in the diagnosis of pneumonia is low (3). Sputum samples and antigen tests may be useful in the diagnosis; however, these methods are time-consuming and lack sensitivity (4).

Biomarkers have the potential to aid health practitioners in clinical practice. The information provided by biomarkers should not duplicate that available by standard methods and the use of the biomarker should enable certain definitive actions to be taken. Prior to the acceptance of a specific biomarker for clinical use, the biomarker should have been proven to be successful in at least one of the following: (i) Showing diagnostic or prognostic efficacy; (ii) reflecting disease progression; (iii) aiding patient selection for specific therapeutic regimens; (iv) exhibiting a wide variety of values; and (v) being unaffected by the duration of the infection and continuing to be show efficacy even during prolonged and successive infections (5).

Aging is often associated with an increase in inflammation and in the risk of community-acquired pneumonia (6). Interleukin-1 receptor antagonist (IL-1RA) is an anti-inflammatory protein that counteracts the destructive effects of inflammatory proteins (7). Taking this into account, it was hypothesized in the present study that pneumonia in elderly patients may be associated with reduced levels of IL-1RA. Tobacco smoking has also been associated with impaired pulmonary function and an increased incidence of infections $(8,9)$, including in elderly patients $(10)$; however, the mechanism behind this phenomenon has yet to be elucidated. Therefore, in the present study, it was also investigated whether smoking affects the level of IL-1RA and increases the risk of pneumonia. 


\section{Materials and methods}

Experimental populations. Ethical approval was obtained from the General Hospital of Shenyang Military Area Command (Shenyang, China) and written informed consent was obtained from the patients (project no. DQKY2011-2049B). Ninety young patients with pneumonia (52 males and 38 females; 30 smokers and 60 non-smokers), aged $\sim 22$ years (range, 18-24 years) and 90 elderly patients with pneumonia (50 males and 40 females; 40 smokers and 50 non-smokers), aged $\sim 70$ years (range, 65-73 years) were selected for this study. Patients with collagen vascular diseases (CVDs) were excluded. For all patients, the diagnosis was pathologically confirmed by open lung biopsy or video-assisted thoracoscopic surgery. The mean \pm standard deviation (SD) percentage vital capacity (\%VC) of the young patients was $88 \pm 25 \%$ (range, $68-120 \%$ ) and the mean $\pm \mathrm{SD}$ arterial oxygen tension $\left(\mathrm{PaO}_{2}\right)$, measured while breathing room air, was $11 \pm 1.9 \mathrm{kPa}$ (range, $8-15 \mathrm{kPa}$ ). The mean \pm SD $\%$ VC of the elderly patients was $85 \pm 16 \%$ (range, 61-112\%) and the mean $\pm \mathrm{SD}^{\mathrm{PaO}_{2}}$ while breathing room air was $11 \pm 1.3 \mathrm{kPa}$ (range, 9-13 $\mathrm{kPa}$ ). In all patients, crackles were present predominantly in basal areas and inspiratory squeaks were not found. No positive serological or other markers of CVD were observed. None of the patients had taken medicine or immunosuppressant therapy at the time of the clinical sample collection.

Blood sample collection and bronchoalveolar lavage fluid $(B A L F)$ procedure. Serum samples were obtained from young and elderly patients. Peripheral venous blood samples were taken and immediately stored at $-80^{\circ} \mathrm{C}$. BAL was performed using a flexible fiberoptic bronchoscope (Olympus 1T-200; Olympus, Tokyo, Japan) following local anesthesia of the upper airways with $4 \%$ lidocaine. The bronchoscope was inserted into one of the segmental bronchi of the right middle lobe and $50 \mathrm{ml}$ sterilized saline was then instilled through the bronchoscope. The fluid was immediately retrieved by suction using a sterile syringe and the procedure was repeated three times. BALF was passed through two sheets of gauze and then centrifuged at $500 \mathrm{xg}$ for $10 \mathrm{~min}$ at $4^{\circ} \mathrm{C}$. The cells were washed twice with phosphate-buffered saline (PBS) and the remaining cells were then suspended in PBS supplemented with $10 \%$ heat-inactivated fetal calf serum and counted using a hemocytometer. An aliquot was then diluted to a concentration of $2 \times 10^{5}$ cells $/ \mathrm{ml}$ and a $0.2-\mathrm{ml}$ cell suspension was spun down onto a glass slide at $300 \mathrm{xg}$ for $2 \mathrm{~min}$. The remaining fluid was centrifuged at $500 \mathrm{xg}$ for $5 \mathrm{~min}$ and the supernatant was stored at $-80^{\circ} \mathrm{C}$. The slides were dried, fixed and then stained using the May-Giemsa method.

Western blot analysis of the relative protein levels of IL-IRA. Antibodies against IL-1RA and GAPDH (loading control) were purchased from Beijing Biotech Co., Ltd., (Beijing, China). BALF and serum were dissolved in radioimmunoprecipitation assay buffer [150 mM sodium chloride, $1 \%$ NP-40, $0.5 \%$ sodium deoxycholate, $0.1 \%$ SDS and $50 \mathrm{mM}$ Tris- $\mathrm{HCl}$ ( $\mathrm{pH}$ 8.0)] and cOmplete Mini protease inhibitor [Roche R\&D Center (China) Ltd., Shanghai, China] was included. The debris was removed and the supernatants were boiled and mixed with an equal volume of $20 \%$ glycerol containing $0.02 \%$ bromophenol blue. Proteins were separated using SDS-PAGE and transferred to a polyvinylidene difluoride membrane (Millipore Corporation, Billerica, MA, USA). The membranes were blocked with $5 \%$ skimmed milk in $10 \mathrm{mM}$ Tris ( $\mathrm{pH} 7.5)$, $100 \mathrm{mM} \mathrm{NaCl}$ and $0.1 \%$ Tween-20 (TBST) and incubated with primary antibodies in TBST with $0.5 \%$ skimmed milk overnight at $4^{\circ} \mathrm{C}$. The membranes were treated with primary antibodies and horseradish peroxidase-conjugated goat secondary antibodies (Sangon Biotech Co., Ltd., Shanghai, China).

Measurement of the immunological activity of IL-1RA. The levels of IL-1RA were measured using commercial kits in accordance with the manufacturer's instructions. IL-1RA concentrations in the serum and BALF were measured using an ELISA kit (Sino Biological Inc., Beijing, China). To compare the levels of IL-1RA with other biomarkers of pneumonia known to be useful for clinical decisions, the serum levels of periostin, a matrix protein used as a biomarker to assess histopathological types of pneumonia, were additionally measured (11). Periostin levels were measured using an ELISA kit (R\&D Systems China Co., Ltd., Beijing, China).

Statistical analysis. Differences between the two groups were compared using one-way analysis of variance. Fisher's protected least significant difference test was used as a post hoc test. Spearman's rank correlation coefficient was used to identify the strength of correlation between the levels of IL-1RA in the serum and BALF. The correlation between tobacco smoking and the levels of IL-1RA was also examined. All subjects had a personal history of smoking 0 to 12 cigarettes/day (the same brand of cigarettes) for the last 10 years. Online software (available at http://www.wessa.net/rwasp_ spearman.wasp/) was used to compute the Spearman's Rank Correlation and the two-sided P-value, as well as to create the ordinary scatterplot between the ranks of $\mathrm{X}$ and Y. Data were analyzed using Statview 5.0 software (Abacus systems, Berkeley, CA, USA). $\mathrm{P}<0.05$ was considered to indicate a statistically significant difference.

\section{Results}

Cell counts in BALF. Numerous types of cell are responsible for producing cytokines in response to local or systemic injury. These cells can be local resident cells within the lung, including alveolar macrophages, lung epithelial cells and fibroblasts, or cells such as neutrophils, lymphocytes, monocytes and platelets (12-14). The number of inflammatory cells may reflect certain characteristics of specific types of pneumonia. For young patients, the total cell counts of macrophages, eosinophils and neutrophils were significantly higher than those in elderly patients $(\mathrm{P}<0.05)$. However, in the elderly patients, the absolute number of lymphocytes was higher than those in the young patients $(\mathrm{P}<0.05)$ (Table I). Thus, pneumonia in elderly patients may be characterized by the high levels of the predominant inflammatory cells, lymphocytes.

Relative protein levels of IL-IRA in the serum and BALF. The relative protein levels of IL-1RA in the serum differed between young and elderly patients: The relative protein levels 
Table I. Cell counts in bronchoalveolar lavage fluid among different groups.

\begin{tabular}{|c|c|c|c|c|c|c|}
\hline Patient groups & Number & Total cells $\mathrm{s}^{\mathrm{a}}, \mathrm{x} 10^{5} / \mathrm{ml}$ & Macrophages $^{\mathrm{a}}$ & Neutrophils ${ }^{\mathrm{a}}$ & Eosinophils $^{\mathrm{a}}$ & Lymphocytes $^{\mathrm{a}}$ \\
\hline Young & 40 & $5.7(11.8)$ & $75.8(36.3)$ & $6.4(5.5)$ & $3.8(6.1)$ & $13.7(8.5)$ \\
\hline Elderly & 50 & $4.4(1.3)$ & $52.8(21.1)$ & $3.1(1.9)$ & $1.7(1.8)$ & $42.0(21.3)$ \\
\hline
\end{tabular}

All values are expressed as means and (medians). ${ }^{\mathrm{a}} \mathrm{P}<0.05$ for the overall comparison of the two groups (one-way analysis of variance).
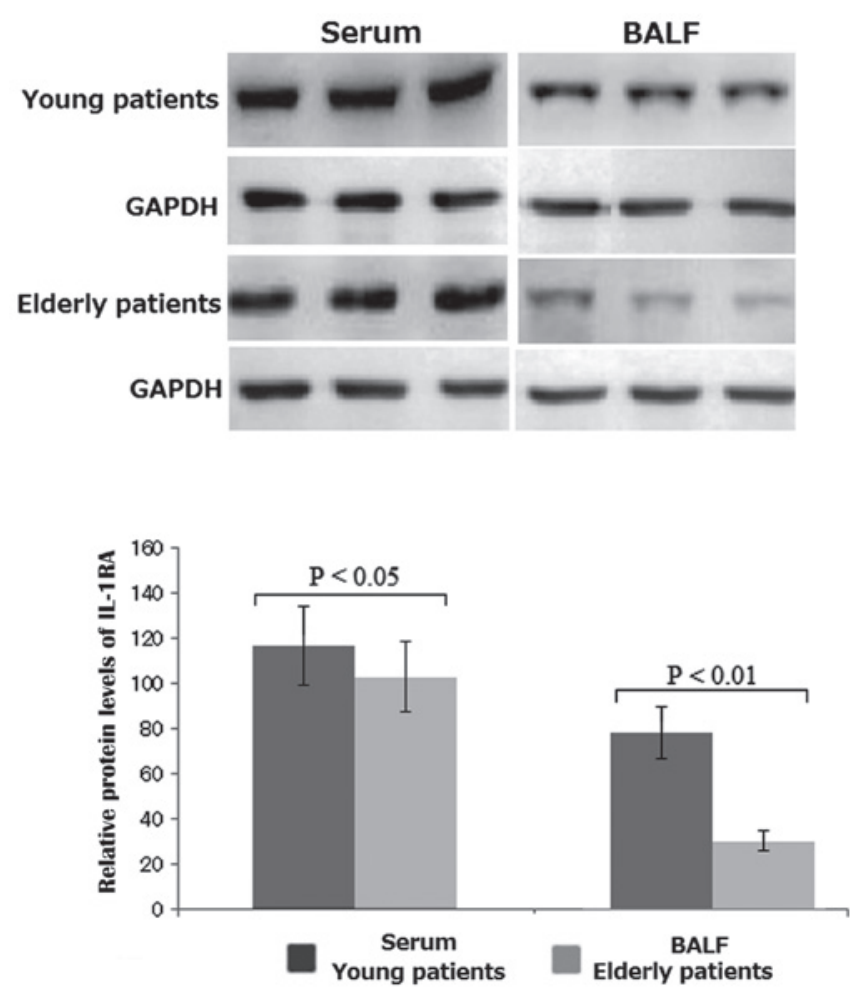

Figure 1. Western blot analysis of the relative protein levels of interleukin-1 receptor antagonist in serum and BALF from young and elderly patients. Each bar represents the mean \pm standard deviation of three independent experiments. BALF, bronchoalveolar lavage fluid; IL-1RA, interleukin-1 receptor antagonist.

of IL-1RA in the serum were lower in elderly patients than those in young patients $(\mathrm{P}<0.05$; Fig. 1$)$. Similarly, the relative protein levels of IL-1RA in BALF differed significantly between young and elderly patients: The relative protein levels of IL-1RA in the BALF were lower in elderly patients than those in young patients $(\mathrm{P}<0.01$; Fig. 1$)$. This difference in protein levels between young and elderly patients was greater in the BALF than that in serum. Therefore, reduced protein levels of IL-1RA in BALF may act as an effective biomarker for the diagnosis of elderly pneumonia.

Immunological activities of IL-1RA in the serum and BALF. The average concentrations of IL-1RA were $160 \mathrm{pg} / \mathrm{ml}$ (range, $84-185 \mathrm{pg} / \mathrm{ml}$ ) and $138 \mathrm{pg} / \mathrm{ml}$ (range, $78-170 \mathrm{pg} / \mathrm{ml}$ ) in the serum of the young and elderly patients, respectively. The average concentrations of IL-1RA were $85 \mathrm{pg} / \mathrm{ml}$ (range, $50-143 \mathrm{pg} / \mathrm{ml}$ ) and $70 \mathrm{pg} / \mathrm{ml}$ (range, 40-105 pg/ml) in the BALF of young and elderly patients, respectively. IL-1RA levels in the serum differed $(\mathrm{P}<0.05)$ between young and elderly patients. The levels of IL-1RA in the serum were higher in young patients

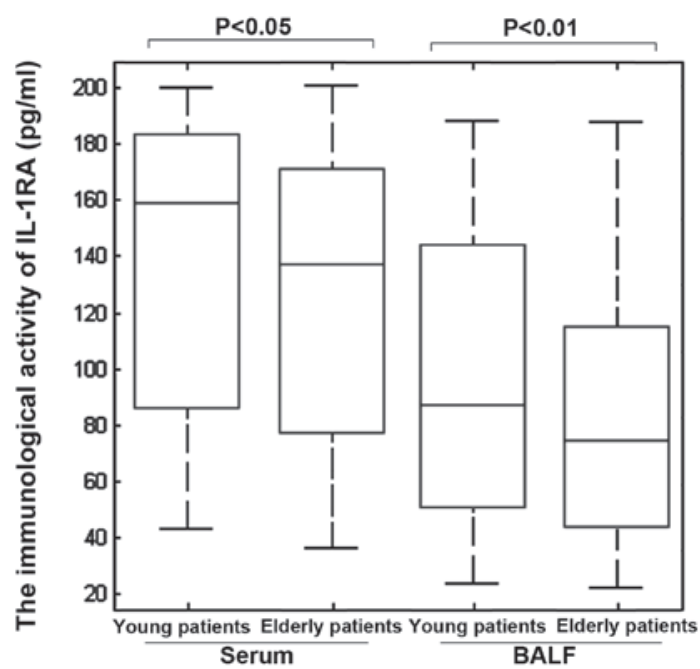

Figure 2. Immunological activities of IL-1RA in the serum and BALF of young and elderly patients. The bars in the boxes are average activities and the boxes represent $95 \%$ of the samples. The error bars are shown above and below the boxes. Young patients, $n=90$; elderly patients, $n=90$. IL-1RA, interleukin-1 receptor antagonist; BALF, bronchoalveolar lavage fluid.

than those in elderly patients (Fig. 2). IL-1RA levels in the BALF also differed significantly $(\mathrm{P}<0.05)$ between young and elderly patients: IL-1RA levels in the BALF were higher in young patients than those in elderly patients (Fig. 2). All the results were in accordance with the results obtained from the western blot analysis.

Correlation between the percentage of lymphocytes and concentration of IL-1RA. The levels of IL-1RA in the serum and the BALF were inversely correlated with the percentage of lymphocytes in all individuals (Fig. 3A and B). No significant correlations were observed between the levels of IL-1RA and the percentages of neutrophils or eosinophils in the BALF. The mean serum concentrations of periostin in young patients were significantly higher than those in elderly patients [young patients, $49 \mathrm{ng} / \mathrm{ml}$ (range, 35.8-77.1 $\mathrm{ng} / \mathrm{ml}$ ) versus elderly patients, $18.7 \mathrm{ng} / \mathrm{ml}$ (range, 11.3-30.1 ng/ml); $\mathrm{P}<0.005$ ] (data not shown). In addition, the clinical utility of reduced serum and BALF IL-1RA levels as markers of pneumonia in the elderly was investigated. No significant differences were identified in the mean $\% \mathrm{VC}$ or $\mathrm{PaO}_{2}$ between young and elderly patients. The serum and BALF levels of IL-1RA also did not significantly correlate with any of the pulmonary function test results or $\mathrm{PaO}_{2}$ (data not shown) in the patients with pneumonia.

Association between smoking and the levels of IL-1RA. Smoking damages the natural defenses of the body against 

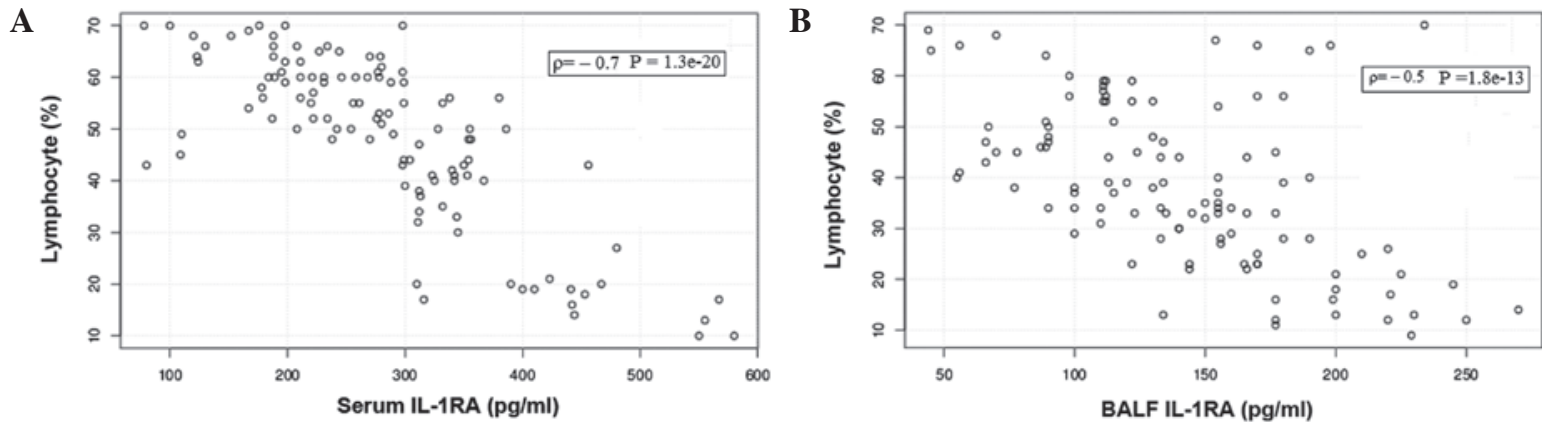

Figure 3. Correlation between the percentage of lymphocytes and IL-1RA levels in (A) serum and (B) BALF from young and elderly patients. Statistical analysis was performed using a Spearman's rank correlation test. Values of $\mathrm{o}$ between -1 and -0.5 indicate a strong negative correlation; values between 0.5 and 1 indicate a weak positive correlation. IL-1RA, interleukin-1 receptor antagonist; BALF, bronchoalveolar lavage fluid.

A

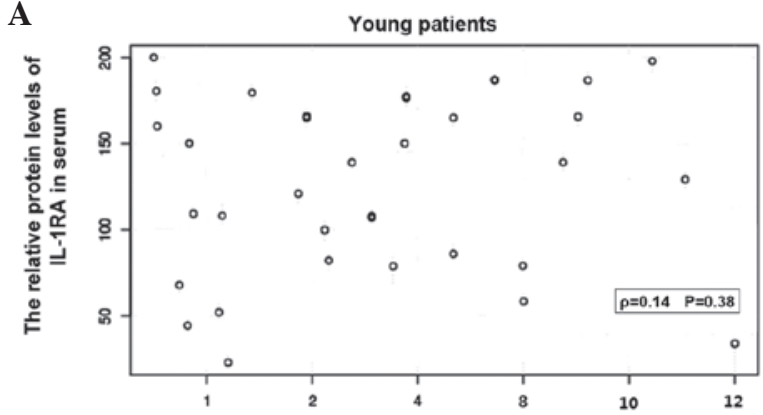

Personal histories of tobacco habit of from 0 to 12 cigarettes/day

C

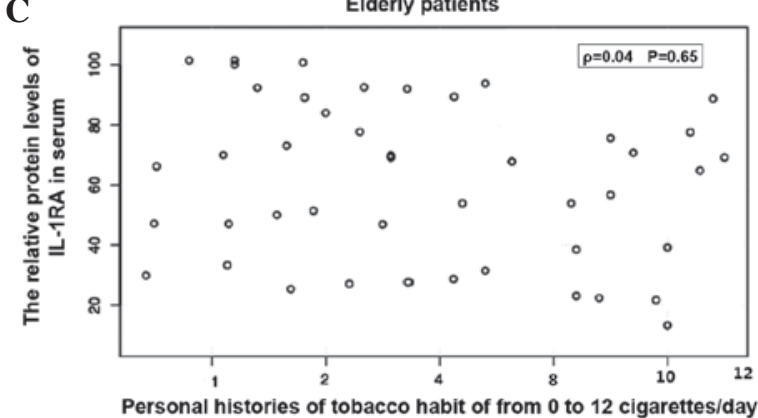

B

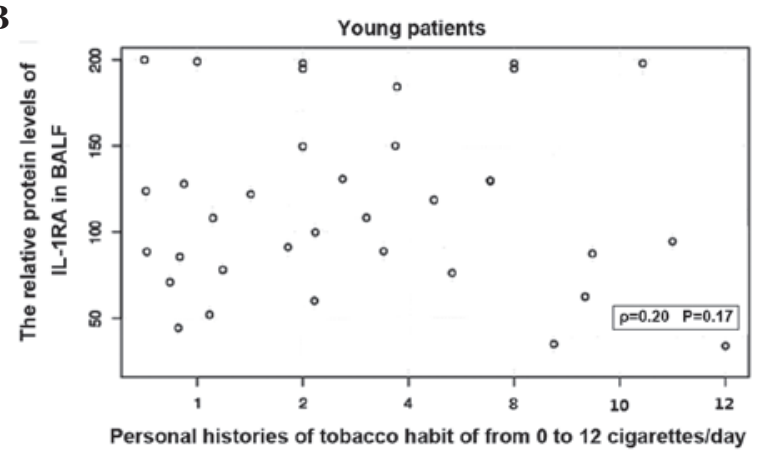

D

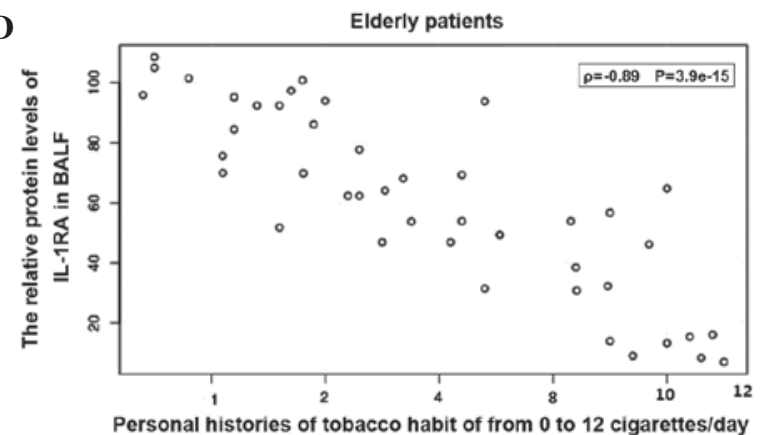

Figure 4. Correlation between tobacco smoking and the expression levels of IL-1RA in the (A) serum from young patients, (B) BALF from young patients, (C) serum from elderly patients and (D) BALF from elderly patients. Values of $\varrho$ between -1 and -0.5 indicate a strong negative correlation; values between 0.5 and 1 indicate a weak positive correlation. IL-1RA, interleukin-1 receptor antagonist; BALF, bronchoalveolar lavage fluid.

bacteria and viruses that increase the risk of hospitalization for pneumonia $(15,16)$. In the present study it was hypothesized that pneumonia in elderly patients may be due to reduced levels of IL-1RA; therefore, it was also investigated whether smoking affected the expression levels of IL-1RA. In young patients, the average concentrations of IL-1RA in serum and BALF were not correlated with the degree of tobacco smoking ( $\mathrm{P}>0.05$; Fig. 4A and B). By contrast, the concentration of IL-1RA in the BALF, but not serum, was closely correlated with the degree of tobacco smoking in elderly patients $(\mathrm{P}<0.01$; Fig. 4C and $\mathrm{D})$. These results suggested that smoking may cause a decrease in the expression levels of IL-1RA in the BALF of elderly patients. The association between the degree of tobacco smoking and the levels of IL-1RA in the BALF indirectly provides support for the hypothesis that smoking may contribute to the pathogenesis of pneumonia in elderly patients by reducing IL-1RA levels in the BALF but not the serum.

\section{Discussion}

This study demonstrated that reduced levels of IL-1RA may lead to an increased number of lymphocytes in the BALF of elderly patients compared with the number of lymphocytes in the BALF of young patients. The levels of IL-1RA were observed to be inversely correlated with the number of lymphocytes in the BALF. These findings suggest that the reduced levels of IL-1RA at local inflammatory sites may lead to the pathogenesis of pneumonia in elderly patients and an increase in the number of lymphocytes in the BALF. Surgical lung biopsy remains an important method for the pathological diagnosis and clinical selection of therapeutic management 
for pneumonia. Clinicians often speculate on the presence of such pathological changes based on non-invasive imaging studies, for example high-resolution computed tomography (HRCT) scans (17-19). However, the discrimination between pneumonias in young and elderly patients, which appear to have different pathogeneses, may not always be accurately predicted by HRCT. BAL has been demonstrated to be a useful method to permit sampling of cells from alveolar areas. In the present study it was found that reduced levels of IL-1RA were associated with an increased risk of pneumonia in elderly patients; this may provide more information for promoting the prognostic significance of young patients and healthy subjects. The effect of IL-1RA on lymphocyte infiltration remains unknown. In the presents study it was indicated that IL-1RA acts as a functional inhibitor of lymphocyte accumulation in lung lesions and that elderly patients exhibit reduced levels of IL-1RA. It was also demonstrated that there was a significant inverse correlation between the levels of IL-1RA in BALF and the serum levels of periostin in young patients and healthy individuals, with serum levels of periostin being significantly higher in elderly patients.

IL-1RA has been found to have a role in the host immune defense in pneumonia. For example, IL-1RA polymorphism is associated with the risk of multidrug-resistant Acinetobacter baumannii-related pneumonia (20). In a study by Patwari et al (21), the presence or absence of the A1 allele in the IL-1RA intron 2 polymorphic site was associated with a decreased or increased risk, respectively, of severe lung injury in community-acquired pneumonia. Severe lung injury was indicated by the requirement for positive pressure ventilation or the development of acute lung injury or acute respiratory distress syndrome (21). IL-1RA levels have been reported to be low in elderly patients. For example, in patients with colorectal cancer undergoing surgery, low preoperative IL-1RA levels were revealed to be associated with postoperative infection. Furthermore, lower IL-1RA levels were commonly observed in elderly patients with low body mass index. These findings suggest that postoperative infection, which is common among elderly individuals who are nutritionally deficient, may be a consequence of a defective immunoinflammatory adaptation system (22). However, levels of IL-1RA have not been considered for the diagnosis of elderly pneumonia. In the present study it was found that the levels of IL-1RA in elderly patients were significantly lower than those in young patients, resulting in high levels of inflammation in elderly patients and making them vulnerable to the disease. The low levels of IL-1RA were also found to be correlated with the disease activity of pneumonia. These results indicate that low levels of IL-1RA in BALF may be used as a sensitive parameter for the diagnosis of pneumonia in the elderly.

Smoking increases the risk of hospitalization for pneumonia, yet the mechanism behind the phenomenon is unknown. In a previous study, gingival crevicular fluid (GCF) levels of the cytokine IL-1RA were analyzed with respect to smoking in patients with moderate to severe periodontal disease. The clinical characteristics, in terms of probing depth and frequency of diseased sites and supragingival plaque, did not differ between smokers and non-smokers, so the observations did not reveal any influence of smoking on the levels of IL-1RA in GCF (23). However, the present study revealed that changes in IL-1RA levels could be detected in BALF, rather than in serum; BAL has been demonstrated to be useful for analyzing cells from the alveolar areas.

In the present study it was demonstrated that IL-1RA levels in the BALF of elderly patients are reduced compared with those in young patients. In addition, IL-1RA levels were shown to significantly correlate with the number of lymphocytes in the BALF. These results suggest that the lymphocytosis in the BALF of elderly patients is associated with the reduced levels of IL-1RA, which may be caused by smoking. From these results, it is concluded that reduced levels of IL-1RA may act as a novel biomarker for elderly pneumonia. Smoking may increase the risk of pneumonia in the elderly by further reducing IL-1RA levels in the BALF.

\section{References}

1. Wilson BZ, Anzueto A, Restrepo MI, Pugh MJ and Mortensen EM: Comparison of two guideline-concordant antimicrobial combinations in elderly patients hospitalized with severe community-acquired pneumonia. Crit Care Med 40: 2310-2314, 2012.

2. Cabre M: Pneumonia in the elderly. Curr Opin Pulm Med 15: 223-229, 2009.

3. Esayag Y, Nikitin I, Bar-Ziv J, et al: Diagnostic value of chest radiographs in bedridden patients suspected of having pneumonia. Am J Med 123: 88.e1-88.e5, 2010.

4. Murdoch DR: Nucleic acid amplification tests for the diagnosis of pneumonia. Clin Infect Dis 36: 1162-1170, 2003.

5. Christ-Crain $M$ and Opal SM: Clinical review: the role of biomarkers in the diagnosis and management of community-acquired pneumonia. Crit Care 14: 203, 2010.

6. Hinojosa E, Boyd AR and Orihuela CJ: Age-associated inflammation and toll-like receptor dysfunction prime the lungs for pneumococcal pneumonia. J Infect Dis 200: 546-554, 2009.

7. Berezin V and Bock E: Antagonists of the interleukin-1 receptor. WO Patent App. PCT/DK2012/000,022. Filed March 14, 2012; issued September 20, 2012.

8. Mani D, Guinee DG Jr and Aboulafia DM: Vanishing lung syndrome and HIV infection: an uncommon yet potentially fatal sequela of cigarette smoking. J Int Assoc Physicians AIDS Care (Chic) 11: 230-233, 2012.

9. Clifford GM, Lise M, Franceschi S, et al; Swiss HIV Cohort Study: Lung cancer in the Swiss HIV Cohort Study: role of smoking, immunodeficiency and pulmonary infection. $\mathrm{Br}$ J Cancer 106: 447-452, 2012.

10. Kikuchi R, Watabe N, Konno T, Mishina N, Sekizawa K and Sasaki H: High incidence of silent aspiration in elderly patients with community-acquired pneumonia. Am J Respir Crit Care Med 150: 251-253, 1994.

11. Okamoto M, Hoshino T, Kitasato Y, et al: Periostin, a matrix protein, is a novel biomarker for idiopathic interstitial pneumonias. Eur Respir J 37: 1119-1127, 2011.

12. Dolinay T, Kim YS, Howrylak J, et al: Inflammasome-regulated cytokines are critical mediators of acute lung injury. Am J Respir Crit Care Med 185: 1225-1234, 2012.

13. Sisson TH, Mendez M, Choi K, et al: Targeted injury of type II alveolar epithelial cells induces pulmonary fibrosis. Am J Respir Crit Care Med 181: 254-263, 2010.

14. Grommes J and Soehnlein O: Contribution of neutrophils to acute lung injury. Mol Med 17: 293-307, 2011.

15. Cecere LM, Williams EC, Sun H, et al: Smoking cessation and the risk of hospitalization for pneumonia. Respir Med 106: 1055-1062, 2012.

16. Katzenstein AL: Smoking-related interstitial fibrosis (SRIF), pathogenesis and treatment of usual interstitial pneumonia (UIP), and transbronchial biopsy in UIP. Mod Pathol 25 (Suppl 1): S68-S78, 2012.

17. Jeong YJ, Lee KS, Chung MP, Han J, Johkoh T and Ichikado K: Chronic hypersensitivity pneumonitis and pulmonary sarcoidosis: differentiation from usual interstitial pneumonia using high-resolution computed tomography. Semin Ultrasound CT MR 35: 47-58, 2014. 
18. Lynch DA and Huckleberry JM: Usual interstitial pneumonia: typical and atypical high-resolution computed tomography features. Semin Ultrasound CT MR 35: 12-23, 2014.

19. Eisman Hidalgo M, Nuñez-Delgado Y and García Espona MÁ: Idiopathic lipoid pneumonia findings in chest high resolution computed tomography. A case report. Arch Bronconeumol 50: 83-84, 2014 (In English and Spanish).

20. Hsu MJ, Lu YC, Hsu YC, Liu WS and Wu WT: Interleukin-1 receptor antagonist gene polymorphism in patients with multidrug-resistant Acinetobacter baumannii-associated pneumonia. Ann Thorac Med 7: 74-77, 2012.
21. Patwari PP, O'Cain P, Goodman DM, et al: Interleukin-1 receptor antagonist intron 2 variable number of tandem repeats polymorphism and respiratory failure in children with community-acquired pneumonia. Pediatr Crit Care Med 9: 553-559, 2008.

22. Miki C, Inoue Y, Toiyama Y, et al: Deficiency in systemic interleukin-1 receptor antagonist production as an operative risk factor in malnourished elderly patients with colorectal carcinoma. Crit Care Med 33: 177-180, 2005.

23. Boström L, Linder LE and Bergström J: Smoking and GCF levels of IL-1beta and IL-1ra in periodontal disease. J Clin Periodontol 27: 250-255, 2000. 